\title{
Synthesis of magnetically frustrated oxides with double perovskite structure
}

\author{
A. Smerechuk ${ }^{1,2}$, R. Morrow ${ }^{1}$, S. Wurmehl ${ }^{1}$, O. Sidletskiy ${ }^{2}$ \\ ${ }^{1}$ Leibniz Institute for Solid-State and Materials Research, IFW-Dresden, 01069 Dresden, Germany, \\ ${ }^{2}$ Institute for Scintillation Materials NAS of Ukraine, 61072 Kharkiv, Ukraine \\ a.smerechuk@ifw-dresden.de
}

Intriguing magnetic behaviour has been studied in perovskite structure type materials with the generic formula $A B \mathrm{O}_{3}$ for decades. Here, $A$ is usually an alkaline earth metal, or a rare earth element, while $\mathrm{B}$ is typically a transition metal. In the related double perovskite structure, two different $B$ cations alternate in a rock salt ordering pattern, leading to the general formula $A_{2} B B^{\prime} \mathrm{O}_{6}[1]$. One of the common features in perovskites is the tilting of the octahedra. As an effect, it tunes the band width of multiple orbitals and the strength and sign of the more typical exchange interactions.

The $B$-site ordered double-perovskite oxides, where $A$ is $\mathrm{Sr}$ or $\mathrm{Ba}, B$ is $\mathrm{Cu}$ and $B^{6}$ is a diamagnetic hexavalent ion, crystallize in a tetragonal structure with short $\mathrm{Cu}-\mathrm{O}$ bonds in the $a b$ plane and long $\mathrm{Cu}-\mathrm{O}$ bonds along the $c$ axis, due to the cooperative Jahn-Teller effect of the octahedrally coordinated $\mathrm{d}^{9} \mathrm{Cu}^{2+}$ ion. While structurally three dimensional, many of these compounds show lowdimensional magnetic properties [2].

The purpose of our study is to find and investigate high degeneracy frustrated magnetically correlated materials. $\mathrm{Sr}_{2} \mathrm{CuTe}_{0.5} \mathrm{~W}_{0.5} \mathrm{O}_{6}$ has recently been reported as a spin-liquid, where the random distribution of Te occupying d-shell on the $\mathrm{W}$ (empty d-shell) position blocks a key superexchange path [3]. So, the main goal of this work was to investigate the nearby phase diagrams with the aim of searching new materials with interesting properties and promising characteristics.
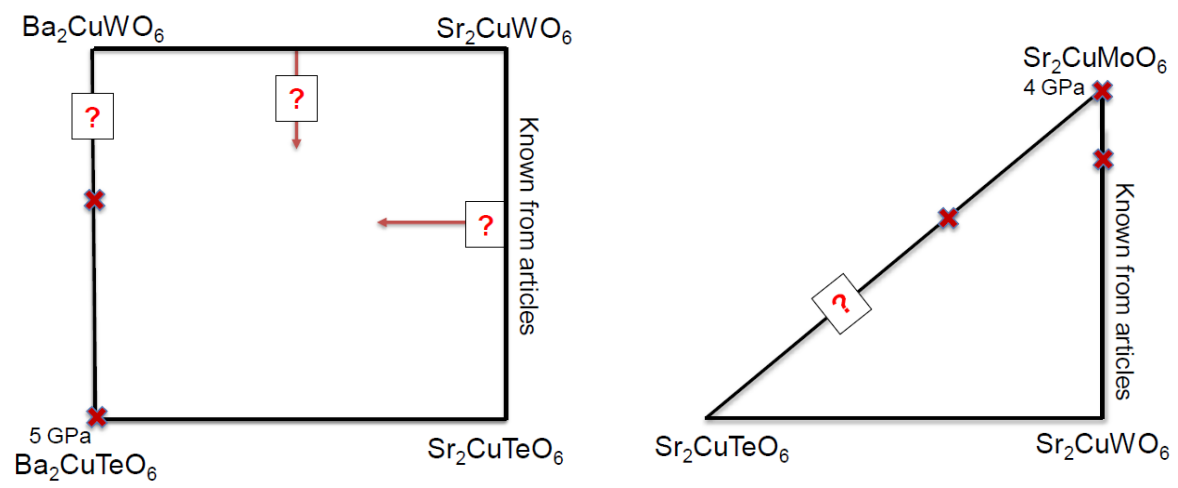

Figure 1. The square and triangle phase diagrams with end members at the edges.

Systematically spaced compositions were attempted in polycrystalline solid state reactions for $\mathrm{Ba}_{\mathrm{x}} \mathrm{Sr}_{2-\mathrm{x}} \mathrm{CuTe}_{0.5} \mathrm{~W}_{0.5} \mathrm{O}_{6}$ and $\mathrm{Sr}_{2} \mathrm{Cu}\left(\mathrm{Te}_{\mathrm{x}} \mathrm{Mo}_{1-\mathrm{x}}\right) \mathrm{O}_{6}$ systems at atmospheric pressure. These efforts were characterized by X-ray diffraction and SQUID magnetometry where successful, and these results as well as future plans will be presented.

[1] Vasala S., Karppinen M. (2015). Prog. Solid State Chem. 43, pp. 1-36.

[2] Todate Y., Higemoto W., Nishiyama K., Hirota K. (2007). J. Phys. and Chem. of Solids, 68, 11, pp. 2107-2110.

[3] Mustonen O., Vasala S., Sadrollahi E., Schmidt K. P., Baines C., Walker H. C., Terasaki I., Litterst F. J., Baggio-Saitovitch E. \& Karppinen M. (2018). Nat. Commun. 9, 1085, pp. 1-8.

Keywords: polycrystals; spin liquids; double perovskites; 2D-magnetism 